\title{
Three-Dimensional Visualization of Shock Cell Structure and Screech Tone Emission in Underexpanded Circular Jet
}

\author{
Keita Ozawa1, Takayuki Yamagata², Nobuyuki Fujisawa ${ }^{2,3}$ \\ ${ }^{1}$ Graduate School of Science and Technology, Niigata University, Niigata, Japan \\ ${ }^{2}$ Department of Mechanical Engineering, Niigata University, Niigata, Japan \\ ${ }^{3}$ Department of Mechanical Engineering, Shinshu University, Nagano, Japan \\ Email: yamagata@eng.niigata-u.ac.jp, fujisawa@eng.niigata-u.ac.jp
}

How to cite this paper: Ozawa, K., Yamagata, T. and Fujisawa, N. (2022) ThreeDimensional Visualization of Shock Cell Structure and Screech Tone Emission in Underexpanded Circular Jet. Journal of Flow Control, Measurement \& Visualization, 10, 1-11.

https://doi.org/10.4236/jfcmv.2022.101001

Received: July 27, 2021

Accepted: October 8, 2021

Published: December 7, 2021

Copyright $\odot 2022$ by author(s) and Scientific Research Publishing Inc. This work is licensed under the Creative Commons Attribution International License (CC BY 4.0).

http://creativecommons.org/licenses/by/4.0/

\begin{abstract}
The three-dimensional visualization of flow and screech-tone emission from an underexpanded circular jet is first investigated experimentally using high-speed cross-schlieren imaging and microphone measurements in the cross-sectional planes along the jet axis. This experimental technique allows the visualization of the shock-cell structure, directivity of sound intensity, and frequency spectrum in the cross-sectional planes of the screeching jet. The high-speed cross-schlieren observation of the screeching jet shows the occurrence of an asymmetrical shock-cell structure that is generated by the flapping mode in the screeching jet. This contributes to the generation of a screech tone propagating upstream along the jet axis and non-uniform sound intensities around the jet in circumferential direction. These observations by high-speed cross-schlieren imaging were validated by the microphone measurements.
\end{abstract}

\section{Keywords}

Screech Tone, Aeroacoustics, Directivity, Frequency Spectrum, Underexpanded Circular Jet, Schlieren Imaging

\section{Introduction}

Since the finding of screech tone by Powell in 1953 [1], this topic of interest has been studied by many researchers, and the results are well summarized in some review papers [2]-[7], which covers a wide range of research from fundamental to applications. It is known that the screech tone is generated by the interaction of the unsteady jet shear layer of high-speed flow and the train of shock cells in 
the jet, where the instability wave propagates downstream and is amplified by the feedback mechanism of the acoustic wave propagating upstream [1] [2]. It has a high-intensity discrete frequency tone in the order of tens of kilohertz in frequency with directivity of tone upstream [2] [3]. Therefore, the generation of screech tone in the jet has a crucial impact on the environmental issue. Because this is the fundamental topic of interest in the field of aeroacoustics, further study on the mechanism of screech-tone generation [8] [9], selection mechanism of oscillation modes [10] [11] [12] [13], and the shock-cell structure in the jet [14] [15] are investigated experimentally. Furthermore, the flow characterizations of screeching jet were performed by introducing the particle image velocimetry [14] and frequency analysis by high-speed schlieren imaging [15]. Note that these characteristics of screech tone were well reproduced in the numerical simulations [16] [17].

To understand the mechanism of the screech tone emission in the jet, schlieren imaging combined with the high-speed flow observation was utilized as a tool of flow visualization for studying the unsteady high-speed flow field, thereby enabling the understanding of the shockwave formation and acoustic sound field in the screeching jet [15]. This experimental technique allows the characterization of an acoustic wave generated in the screeching jet, and the screech tone is emitted toward the upstream, indicating the directivity of the sound in the plane along the jet axis upstream. The shock-cell structure in the jet shear layer starts oscillation normal to the jet axis at fourth to fifth shock cells to generate the acoustic waves of a screech tone [3]. It should be mentioned that these previous studies pertaining to the acoustic field of the screeching circular jet are limited to the axial plane, which shows the screech tone emission directing upstream [2] [3]. However, the three-dimensional behavior of the acoustic field in the circumferential direction normal to the axial plane has not been investigated in the literature because of the experimental challenge.

In this paper, a three-dimensional acoustic field of a screeching circular jet was first investigated experimentally based on high-speed cross-schlieren imaging and microphone measurements around the screeching jet. Furthermore, the circumferential observations of the screech tone emission and frequency spectrum were evaluated experimentally. This enables the first observation of the acoustic behavior of the screech tone in the circumferential plane normal to the jet axis of the underexpanded circular jet.

\section{Experimental Apparatus and Method}

Figure 1 shows the experimental setup for investigating an underexpanded air jet issued from a circular converging nozzle into a still atmospheric environment comprising air. This flow configuration shows a choked flow in the nozzle. Compressed air was provided from a compressor operating up to $P_{0}=0.4 \mathrm{MPa}$ and passed through a filter, valve, and flow meter, which were connected by a pipe of inner diameter $7.5 \mathrm{~mm}$ and discharged into the atmospheric air environment at $P_{a}=0.1 \mathrm{MPa}$. Therefore, the pressure ratio $P_{0} / P_{a}=4$, which corresponds to the 


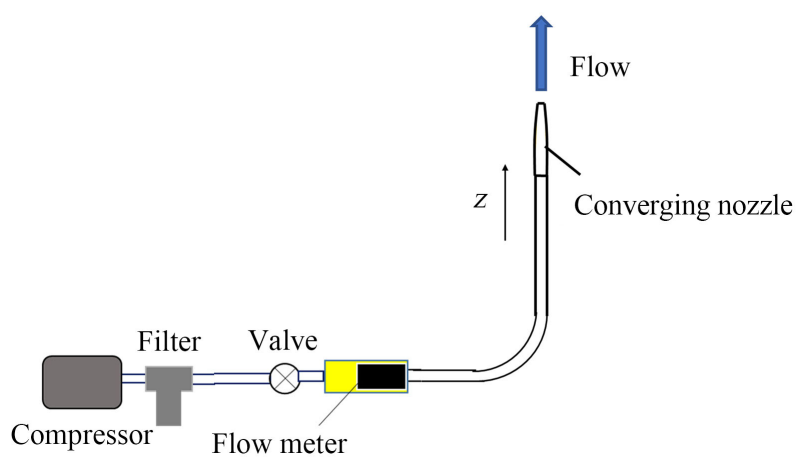

Figure 1. Experimental setup for screeching jet.

fully expanded jet Mach number $M_{\mathrm{j}}=1.56$. A converging nozzle with a circular exit cross-section of diameter $d=3 \mathrm{~mm}$ was attached downstream of the pipe and the flow spreads in vertical direction ( $z$ direction) in an atmospheric environment to facilitate the cross-schlieren imaging along the jet axis. The pressure ratio of the nozzle exiting the atmospheric environment can be varied to generate an underexpanded circular jet downstream of the nozzle. It is noteworthy that the mass flow rate of air was set to $0.36 \mathrm{~kg} / \mathrm{min}$, as measured using a digital flow meter, at pressure ratio 4 for the schlieren imaging and microphone measurement, whereas the microphone measurement was conducted at a lower mass flow rate $0.18 \mathrm{~kg} / \mathrm{min}$ at low pressure ratio 2 for comparative purpose. The sound pressure level (SPL $[\mathrm{dB}]$ ) was detected by a $1 / 2$ inches microphone with a flat frequency response up to $100 \mathrm{kHz}$, and the sampled data after converting to the digital data were analyzed to measure the SPL and frequency spectrum using the fast Fourier transformation algorithm.

Figure 2 shows the experimental setup, which comprises two sets of high-speed schlieren systems equipped with light-emitting diodes (LEDs) with $700 \mathrm{~lm}$ for illumination, concave mirrors, and high-speed complementary metal-oxide semiconductor (CMOS) cameras with camera lenses of focal length 200 $\mathrm{mm}$. It is noteworthy that these schlieren systems are equipped in horizontal plane ( $x-y$ plane) to observe the vertical screeching jet in still air. The diameter and focal length of the concave mirror were 150 and $1200 \mathrm{~mm}$, respectively, and the cameras were operating at 100,000 frames/s at a spatial resolution of $256 \times$ 160 pixels for a sampling period of $0.4 \mathrm{~s}$. These cameras were placed in orthogonal directions along the jet axis in the horizontal plane, and they were parallel with the LED lights through the concave mirrors and pin-hole type knife-edges, which were placed in front of each camera to increase the sensitivity of the schlieren images [18] [19]. The diameter of the pin hole was $1 \mathrm{~mm}$. The shutter speed of the camera was set to $250 \mathrm{~ns}$, which was the shortest exposure time of the camera, whereas a higher shutter speed might be better suited for capturing high-speed flow events. The two CMOS cameras were operated synchronously using a pulse controller. It is noteworthy that schlieren images 1 in the $x$ - $Z$ plane and schlieren images 2 in the $y-z$ plane were captured by cameras 1 and 2 , respectively, as illustrated in Figure 2. 


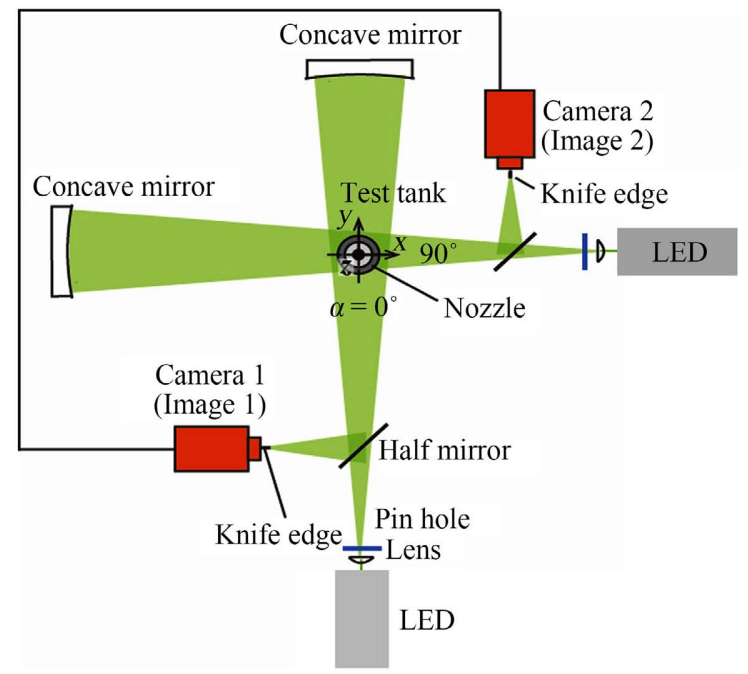

Figure 2. High-speed cross-schlieren imaging system (Top view).

Image processing and enhancement of schlieren images were performed using contrast correction, edge enhancement, and noise reduction [15]. To eliminate the imperfections of the images, no-flow image was subtracted from the original schlieren images. The frequency spectrum was obtained from these images by introducing the fast-Fourier-transformation analysis at the sensing position in the images.

\section{Results and Discussion}

\subsection{Three-Dimensional Flow Field of Circular Screeching Jet}

To confirm the formation of an axisymmetric flow field in the screeching circular jet at pressure ratio $P_{0} / P_{a}=4$, the total pressure distributions were measured by traversing the total pressure tube with $1 \mathrm{~mm}$ inner diameter along the $x$ - and $y$-axes normal to the jet axis at several vertical positions $z / d$, where $z$ is the vertical distance from the nozzle exit, and $d$ is the nozzle exit diameter. Note that the uncertainty of the pressure measurement is $1 \%$, and that of the position measurement is $0.1 \mathrm{~mm}$. The experimental results are shown in Figure 3 for several vertical positions $z / d=0.3-6.3$. It is noteworthy that $P_{t}$ is the total pressure, and $P_{t 0}$ is the total pressure at the nozzle exit. Although the centerline pressure decreased immediately at a short distance from the nozzle, it recovered downstream, and the total pressure distribution exhibited a maximum at the jet centerline downstream. This indicates the peculiar feature of the screeching jet near the nozzle exit down to $z / d=5$, which was caused by the compressible air jet. It is expected that the decrease in total pressure at immediate downstream of nozzle exit $(z / d=1.3)$ can be caused by the effect of jet expansion to the outside of the jet axis of the underexpanded jet and the resulted low velocity along the jet centerline. However, the total pressure distributions remained axisymmetric with respect to the jet axis, suggesting the formation of an axisymmetric flow field in the screeching circular jet. Note that the scattering of the data was smaller than the size of the marks in Figure 3. 


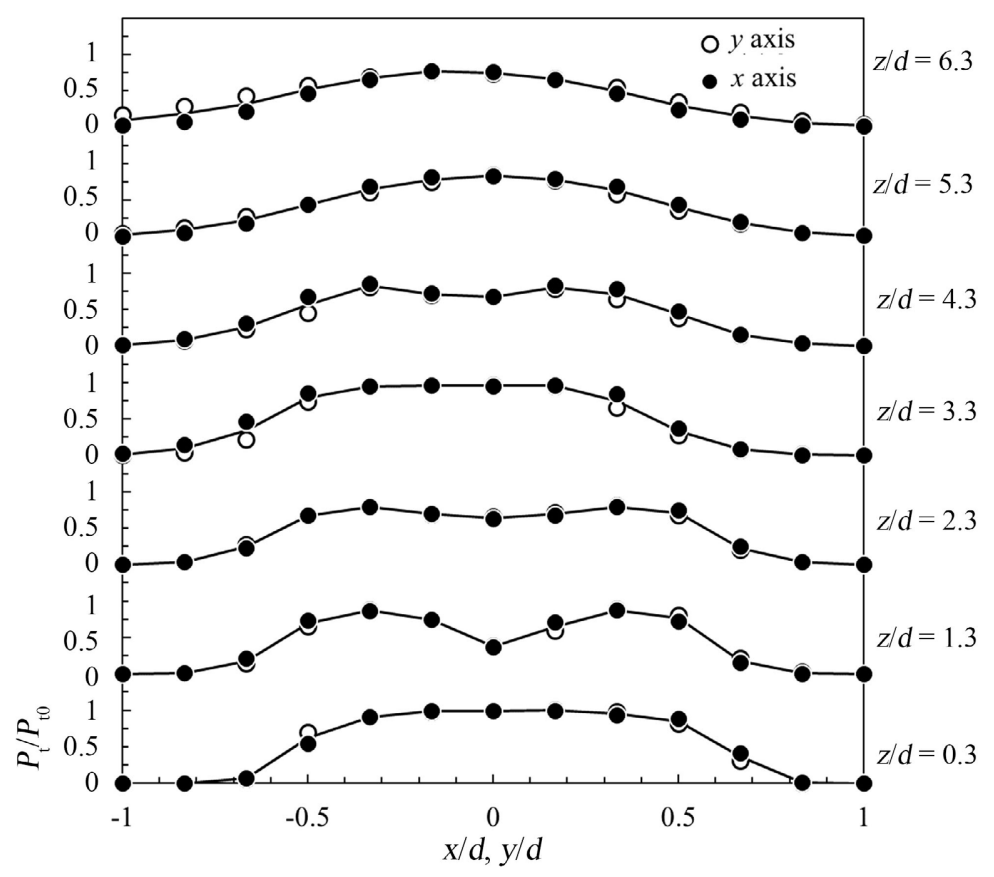

Figure 3. Cross-sectional total pressure distributions at several downstream positions $z / d$.

\subsection{High-Speed Cross-Schlieren Imaging}

Figure 4 shows three examples of high-speed cross-schlieren images 1 in the $x-Z$ plane and images 2 in the $y$ - $z$ plane, which were captured by cameras 1 and 2, respectively, of the underexpanded circular air jet issuing into the still air environment. Note that time interval of each sequential image is set to $10 \mu \mathrm{s}$. It is noteworthy that the black circle in the picture was caused by the center mark printed on the mirror surface in advance. Both schlieren images showed shock-cell structures along the shear layer downstream of the nozzle. Schlieren images 1 showed a staggered arrangement of the shock-cell structure downstream of the jet, whereas schlieren images 2 showed a shock-cell structure that developed straight along the jet axis in $z$ direction. Hence, a set of cross-schlieren images showed the formation of a flapping mode, which could be caused by a pair of helical modes of shock cell structures downstream of the nozzle along the jet axis [12]. The shock-cell structure was extremely distorted at the fourth to fifth cell by the formation of flapping mode. The position of distortion is at $z / d=$ 6 from the nozzle in agreement with the previous observation [3].

Therefore, the screech tone can be generated radially from the position $z / d=6$ along jet axis directing far upstream, as evident by the high intensity variation of acoustic waves upstream in schlieren images 1 on the left-hand-side of the image, but not clearly visible in schlieren images 2 .

Figure 5 shows the corresponding cross-schlieren images 1 and 2 in the underexpanded circular jet after image processing and enhancement. These images show the formation of acoustic waves propagating upstream from the position $z / d=6$ along the jet centerline in the underexpanded circular jet. However, the 


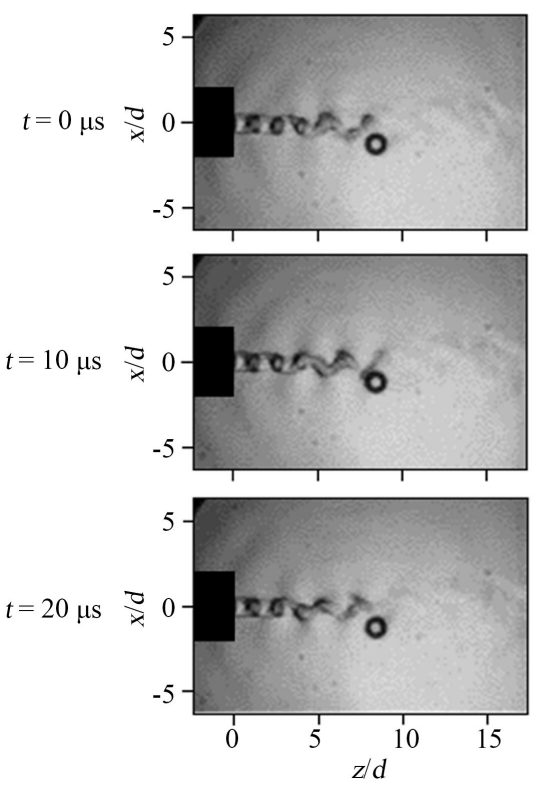

(a) Schlieren images 1
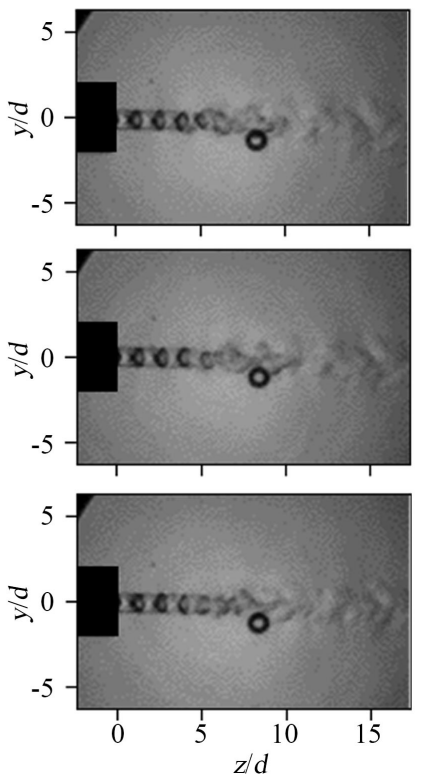

(b) Schlieren images 2

Figure 4. High-speed cross-schlieren images of screeching jet (direct images).

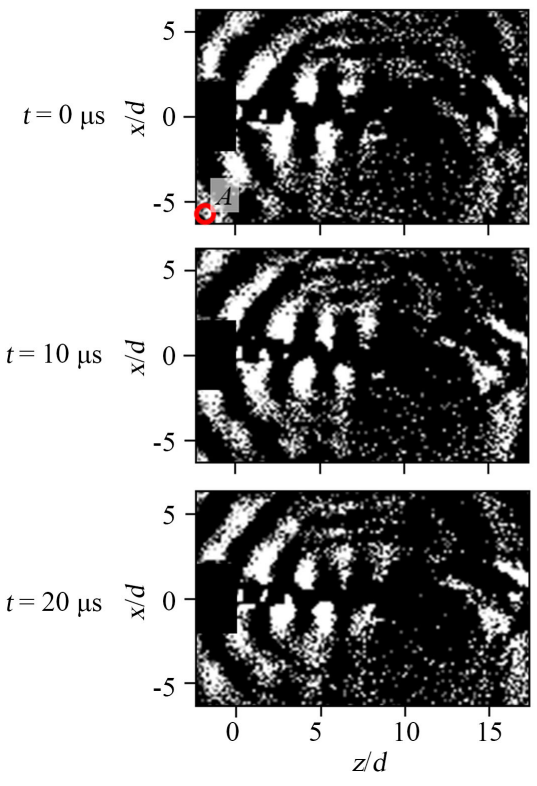

(a) Schlieren images 1
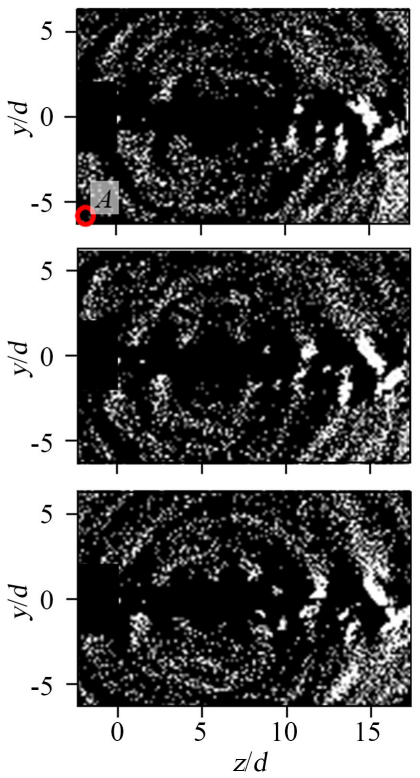

(b) Schlieren images 2

Figure 5. Analyzed schlieren images of screeching jet after image processing (A: sensing position).

intensity distribution of schlieren images 1 and 2 near the nozzle exit indicated a clear difference, whereas the intensity variations were similar in the downstream, i.e., images 1 showed a higher intensity contour near the nozzle than images 2 . This result indicates that high-amplitude acoustic waves were generated in images 1 and were directed upstream consistent with the observation in the literature [2] [3], whereas the magnitude was lower in image 2 near the nozzle. This result suggests that the directivity of the acoustic waves was generated not only 
in the axial plane, but also in the circumferential plane of the underexpanded circular jet, which might result in different noise levels around the jet axis. It should be noted that this result is only available by the cross-schlieren imaging on two orthogonal planes of observation.

\subsection{Frequency Spectra and SPL Measurements}

Figure 6(a) and Figure 6(b) show the frequency spectra obtained from time-series schlieren images 1 and 2, respectively, and they are compared with those of the microphone measurements. The frequency spectra in the schlieren images were obtained at sensing position A marked by red circle in Figure 5. This corresponds to the high intensity of the acoustic waves along the line of $\theta=$ $-135^{\circ}$ at a distance $30 \mathrm{~mm}$ from the position $(z / d=6)$ along the jet axis. Meanwhile, the microphone measurements were performed at the same line of $\theta=$ $-135^{\circ}$ but at a different distance $200 \mathrm{~mm}$ from the position $(z / d=6)$ to minimize the physical interaction with the screeching jet. It is noteworthy that the intensity amplitudes obtained from the schlieren images are shown as arbitrary unit (a.u.) herein because of the difference in schlieren image intensities arising from the minor knife-edge effects and camera settings. However, the relative magnitude of the intensity peaks with respect to the surrounding noise level in each image might not be sensitive to the experimental setting. A comparative study of the frequency spectra of schlieren images 1 and 2 showed a substantial difference in the spectral distributions. Both results showed high peaks at the same frequency $(f=42 \mathrm{kHz})$, but the peak amplitude was higher in the spectrum of schlieren images 1 than that of schlieren images 2 . This result reflects the higher intensity amplitude of the screech tone in schlieren images 1 than that in images 2, suggesting the circumferential directivity of the screech tone in the underexpanded circular jet. Furthermore, the second peak was observed at 17 $\mathrm{kHz}$ in the spectrum of schlieren images 1 , although it did not appear in the spectrum of schlieren images 2 .

To validate the different features of the frequency spectrum captured in schlieren images 1 and 2, further microphone measurements were performed, and the results were compared with the schlieren results shown in Figure 6. The frequency spectrum obtained from microphone in $x-Z$ plane showed a high peak at $42 \mathrm{kHz}$, as shown in Figure 6(a), whereas the result from the microphone in $y-z$ plane showed a small peak at $42 \mathrm{kHz}$, as shown in Figure 6(b). This peak frequency agrees with that obtained from the schlieren-image analysis. Therefore, the main features of the frequency spectrum obtained from the schlieren images were well reproduced in the microphone measurements. However, the second peak at $30 \mathrm{kHz}$ in the microphone measurements deviated from that at $17 \mathrm{kHz}$ in the schlieren-image analysis shown in Figure 6(a). This is likely owing to the effects of different sensing positions of the acoustic measurements.

To confirm the directivity of the screech tone from the underexpanded circular jet in the circumferential plane, SPL measurements were conducted for various circumferential angles $\alpha$ in the cross-sectional plane using microphone 


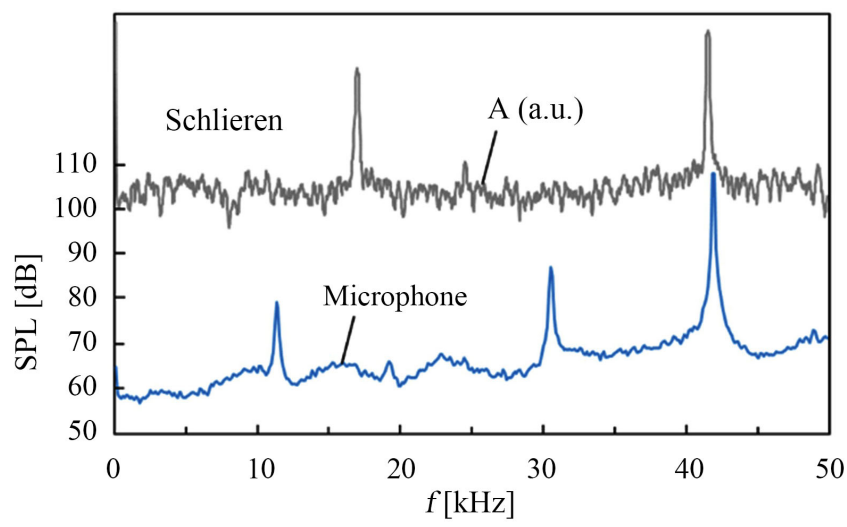

(a) Frequency spectra in $X-Z$ plane $\left(\theta=-135^{\circ}\right)$

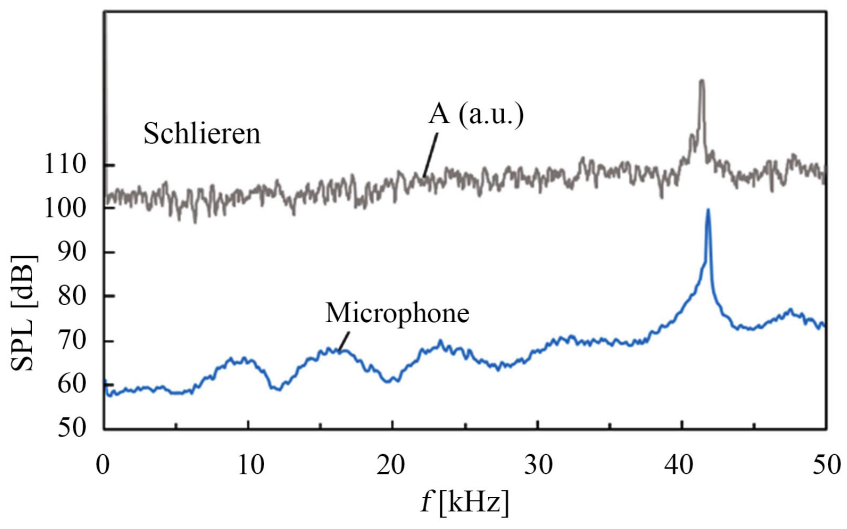

(b) Frequency spectra in $y$ - $z$ plane $\left(\theta=-135^{\circ}\right)$

Figure 6. Frequency spectra in orthogonal cross-sectional planes (A: sensing position, a.u.: arbitrary unit).

measurements at a fixed distance $200 \mathrm{~mm}$ from the jet axis and the angle $\theta=$ $-135^{\circ}$. Figure 7 shows the measured SPL distribution for various circumferential angles $\alpha$. The error bars indicate the scattering of the repeated experimental data. The maximum SPL $(=122 \mathrm{~dB})$ was observed at $\alpha=90^{\circ}\left(270^{\circ}\right)$ in $x-z$ plane, and the minimum SPL $(=110 \mathrm{~dB})$ was recorded at $\alpha=0^{\circ}\left(180^{\circ}\right)$ in $y-z$ plane. Note that the difference of maximum and minimum of SPL is larger than the scattering of the data. The maximum SPL occurs at the staggered arrangement of shock cell structure (Figure 4(a)). Meanwhile, the SPL distribution at a lower pressure ratio of 2 is shown in Figure 7 for comparative purposes. An almost uniform SPL distribution is shown at this pressure ratio, independent of the circumferential angles $\alpha$. However, the SPL distribution at a higher pressure ratio indicates an asymmetrical distribution in the circumferential plane.

This result indicates that an asymmetrical sound field was generated from the circular jet at the pressure ratio 4 , and the screech tone was observed. Therefore, the asymmetry of the sound field is closely related to the screech tone generation. It is noteworthy that the asymmetry of the sound field of the screeching jet can be caused by the flapping mode due to a pair of helical modes, as shown in Figure 4. This may be supported by the results of direct numerical simulation of 


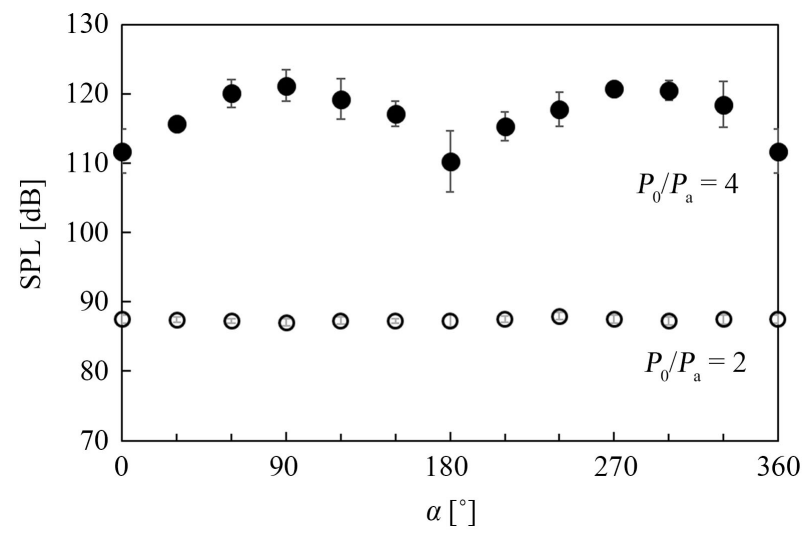

Figure 7. SPL distributions with respect to circumferential angles $\alpha$ by microphone measurements.

a supersonic jet [20] [21], which shows an asymmetric sound field caused by the small disturbance of a pair of helical modes in the upstream, thereby resulting in a flapping mode. However, the selection mechanism of the flapping mode was unclear, and more studies pertaining to the mechanism of the circumferential directivity of the screeching jet noise should be performed in the future.

\section{Conclusion}

The three-dimensional behavior of an acoustic field in a screeching underexpanded circular jet was first investigated using high-speed cross-schlieren imaging and microphone measurement. The experimental technique allowed the visualization of shock-cell structure, directivity of sound, and frequency spectrum of the screeching jet through an analysis of sequential schlieren images captured by two high-speed cameras and microphone measurement. The experimental result indicated the directivity of the acoustic waves upstream, which is consistent with the previous studies in the literature. Furthermore, the cross-schlieren imaging showed that the shock-cell structure became asymmetrical in circumferential plane with respect to the jet axis. Then, the sound intensity distribution became asymmetrical in the circumferential plane, and the peak frequency of the screech tone was observed in both the schlieren images and microphone measurement. The observed asymmetrical sound field in the circumferential plane of the screeching jet could be caused by the occurrence of flapping mode of shock-cell structure arising from a pair of helical-mode disturbances.

\section{Acknowledgements}

The authors would like to thank Prof. D. Watanabe from Toyama University for the helpful communication.

\section{Conflicts of Interest}

The authors declare no conflicts of interest regarding the publication of this paper. 


\section{References}

[1] Powell, A. (1953) On the Mechanism of Choked Jet Noise. Proceedings of the Physical Society, 66, 1039-1056. https://doi.org/10.1088/0370-1301/66/12/306

[2] Seiner, J.M. (1984) Advances in High Speed Jet Aeroacoustics. AIAA Paper, 84-2275. https://doi.org/10.2514/6.1984-2275

[3] Tam, C.K.W. (1995) Supersonic Jet Noise. Annual Review of Fluid Mechanics, 17, 17-43. https://doi.org/10.1146/annurev.fl.27.010195.000313

[4] Matsuo, K., Miyazato, Y. and Kim, H.D. (1999) Shock Train and Pseudo-Shock Phenomena in Internal Gas Flows. Progress in Aerospace Sciences, 35, 33-100. https://doi.org/10.1016/S0376-0421(98)00011-6

[5] Raman, G. (1999) Supersonic Jet Screech: Half-Century from Powell to the Present. Journal of Sound and Vibration, 225, 543-571. https://doi.org/10.1006/jsvi.1999.2181

[6] Bailly, C. and Fujii, K. (2016) High-Speed Jet Noise. Mechanical Engineering Reviews, 3, Article No. 15-00496. https://doi.org/10.1299/mer.15-00496

[7] Edgington-Mitchell, D. (2019) Aeroacoustic Resonance and Self-Excitation in Screeching and Impinging Supersonic Jets-A Review. International Journal of Aeroacoustics, 18, 118-188. https://doi.org/10.1177/1475472X19834521

[8] Tam, C.K.W. (1988) The Shock-Cell Structures and Screech Tone Frequency of Rectangular Non-Axisymmetric Supersonic Jets. Journal of Sound and Vibration, 121, 135-147. https://doi.org/10.1016/S0022-460X(88)80066-X

[9] Panda, J. (1998) Shock Oscillation in Underexpanded Screeching Jets. Journal of Fluid Mechanics, 363, 173-198. https://doi.org/10.1017/S0022112098008842

[10] Powell, A., Umeda, Y. and Ishii, R. (1992) Observations of the Oscillation Modes of Choked Circular Jets. The Journal of the Acoustical Society of America, 92, 2823-2836. https://doi.org/10.1121/1.404398

[11] Ponton, M.K. and Seiner, J.M. (1995) Acoustic Study of B Helical Mode for Choked Axisymmetric Nozzle. AIAA Journal, 33, 413-420. https://doi.org/10.2514/3.12402

[12] Umeda, Y. and Ishii, R. (2002) Sound Sources of Screech Tone Radiated from Circular Supersonic Jet Oscillating in the Helical Mode. Aeroacoustics, 1, 355-384. https://doi.org/10.1260/147547202765275961

[13] Gojon, R., Bogey, C. and Mihaescu, M. (2018) Oscillation Modes in Screeching Jets. AIAA Journal, 56, 2918-2924. https://doi.org/10.2514/1.J056936

[14] Magstadt, A.S., Berry, M.G., Berger, Z.P., Shea, P.R., Ruscher, C.J., Gogineni, S.P. and Glauser, M.N. (2017) Flow Structures Associated with Turbulent Mixing Noise and Screech Tones in Axisymmetric Jets. Flow, Turbulence and Combustion, 98, 725-750. https://doi.org/10.1007/s10494-016-9784-8

[15] Jagadeesh, C., Gowree, E.R., Jadidbonab, H. and Atkin, C.J. (2018) On the Temporal Analysis of Acoustic Waves Using Schlieren Imaging. Measurement, 116, 507-513. https://doi.org/10.1016/j.measurement.2017.11.018

[16] Li, X.D. and Gao, J.H. (2005) Numerical Simulation of the Generation Mechanism of Axisymmetric Supersonic Jet Screech Tones. Physics of Fluids, 17, Article ID: 085105. https://doi.org/10.1063/1.2033909

[17] Singh, A. and Chatterjee, A. (2007) Numerical Prediction of Supersonic Jet Screech Frequency. Shock Waves, 17, 263-272. https://doi.org/10.1007/s00193-007-0110-1

[18] Fujisawa, N., Fujita, Y., Yanagisawa, K., Fujisawa, K. and Yamagata, T. (2018) Simultaneous Observation of Cavitation Collapse and Shock Wave Formation in Ca- 
vitating Jet. Experimental Thermal and Fluid Science, 94, 159-167. https://doi.org/10.1016/j.expthermflusci.2018.02.012

[19] Fujisawa, N., Horiuchi, T., Fujisawa, K. and Yamagata, T. (2018) Experimental Observation of the Erosion Pattern, Pits, and Shockwave Formation in a Cavitating Jet. Wear, 418-419, 265-272. https://doi.org/10.1016/j.wear.2018.10.014

[20] Watanabe, D. and Maekawa, H. (2014) The Effect of Upstream Disturbance on the Angle of Sound Emission in a Supersonic Round Jet. Journal of Fluid Science and Technology, 9, Article No. 14-00181. https://doi.org/10.1299/jfst.2014jfst0054

[21] Watanabe, D. and Maekawa, H. (2015) Transitional Structures and Sound Emission in a Supersonic Round Jet Forced by a Pair of Helical Modes. Proceedings of 9 th International Symposium on Turbulence Shear Flow Phenomenon, Melbourne, 30 June-3 July 2015, Paper 49.

\section{Nomenclature}

A sensing position

$d$ nozzle diameter

$f$ frequency

$M_{\mathrm{j}}$ fully expanded jet Mach number

$P_{t}$ total pressure

$P_{t 0}$ total pressure at nozzle exit

$P_{a}$ pressure in surrounding air

$P_{0}$ pressure upstream

SPL sound pressure level

$x, y$ coordinates normal to jet axis

$z$ coordinate along jet axis

$\alpha$ angle in circumferential plane

$\theta$ angle in axial plane 